International Journal of Pure and Applied Mathematics

Volume 94 No. 2 2014, 215-221

ISSN: 1311-8080 (printed version); ISSN: 1314-3395 (on-line version)

url: http://www.ijpam.eu

doi: http://dx.doi.org/10.12732/ijpam.v94i2.8

ijpam.eu

\title{
CONFORMABLE FRACTIONAL HEAT DIFFERENTIAL EQUATION
}

\author{
M. Abu Hammad ${ }^{1}$, R. Khalil ${ }^{2} \S$ \\ ${ }^{1,2}$ Department of Mathematics \\ The University of Jordan \\ Amman, JORDAN
}

Abstract: In this paper, we try to give exact solution of the heat conformable fractional differential equation. Some other differential equations are discussed.

AMS Subject Classification: 26A33

Key Words: heat equation, conformable fractional derivative

\section{Introduction}

Fractional differential equations turned to be successful models of real life phenomenon (see e.g. $[1,2,4,5,6]$ ). This gives one motivation to study and discuss some of the well known classical differential equations, when some of the derivatives are replaced by fractional derivatives. One of the classical equations is the heat equation

$$
\frac{\partial u(x, t)}{\partial t}=\frac{\partial^{2} u(x, t)}{\partial x^{2}}
$$

There are many fractional forms of the heat equation. One of such forms is $\frac{\partial^{\alpha} u(x, t)}{\partial t^{4}}=\frac{\partial^{2} u(x, t)}{\partial x^{2}}, 0<\alpha<1$. Other forms are

$$
\frac{\partial^{\alpha} u(x, t)}{\partial t^{\alpha}}=\frac{\partial^{2} u(x, t)}{\partial x^{2}},
$$

$1 \leq \alpha<2$, and

Received: March 23, 2014

(C) 2014 Academic Publications, Ltd.

${ }^{\S}$ Correspondence author 


$$
\frac{\partial u(x, t)}{\partial t}=\frac{\partial^{\alpha} u(x, t)}{\partial x^{\alpha}},
$$

$0<\alpha<1$.

The heat differential equation was treated for the classical fractional derivatives either Caputo or Riemann definitions.

In this paper we investigate the differential equation:

$$
\frac{\partial^{\alpha}}{\partial t^{\alpha}} \frac{\partial^{\alpha} u(x, t)}{\partial t^{\alpha}}=\frac{\partial^{2} u(x, t)}{\partial x^{2}}, \quad 0<\alpha<1,
$$

with conditions

$$
\begin{aligned}
& u(0, t)=0, \quad t>, \\
& u(1, t)=0, \quad t>0, \\
& \frac{\partial u(x, 0)}{\partial t}=0, \\
& u(x, 0)=f(x), \quad 0<x<1 .
\end{aligned}
$$

where the fractional derivative is the conformable fractional derivative, see [3].

Fractional derivative is as old as calculus. The most popular definitions are:

(i) Riemann-Liouville Definition: If $n$ is a positive integer and $\alpha \in[n-1, n)$, the $\alpha^{\text {th }}$ derivative of $f$ is given by

$$
D_{a}^{\alpha}(f)(t)=\frac{1}{\Gamma(n-\alpha)} \frac{d^{n}}{d t^{n}} \int_{a}^{t} \frac{f(x)}{(t-x)^{\alpha-n+1}} d x .
$$

(ii) Caputo Definition. For $\alpha \in[n-1, n)$, the $\alpha$ derivative of $f$ is

$$
D_{a}^{\alpha}(f)(t)=\frac{1}{\Gamma(n-\alpha)} \int_{a}^{t} \frac{f^{(n)}(x)}{(t-x)^{\alpha-n+1}} d x .
$$

Now, all definitions are attempted to satisfy the usual properties of the standard derivative. The only property inherited by all definitions of fractional derivative is the linearity property. However, the following are the setbacks of one definition or another: 
(i) The Riemann-Liouville derivative does not satisfy $D_{a}^{\alpha}(1)=0\left(D_{a}^{\alpha}(1)=0\right.$ for the Caputo derivative), if $\alpha$ is not a natural number.

(ii) All fractional derivatives do not satisfy the known product rule

$$
D_{a}^{\alpha}(f g)=f D_{a}^{\alpha}(g)+g D_{a}^{\alpha}(f) .
$$

(iii) All fractional derivatives do not satisfy the known quotient rule:

$$
D_{a}^{\alpha}(f / g)=\frac{g D_{a}^{\alpha}(f)-f D_{a}^{\alpha}(g)}{g^{2}} .
$$

(iv) All fractional derivatives do not satisfy the chain rule:

$$
D_{a}^{\alpha}(f \circ g)(t)=f^{(\alpha)}(g(t)) g^{(\alpha)}(t) .
$$

(v) All fractional derivatives do not satisfy: $D^{\alpha} D^{\beta} f=D^{\alpha+\beta} f$ in general.

(vi) Caputo definition assumes that the function $f$ is differentiable.

Authors, introduced a new definition of fractional derivative as follows:

For $\alpha \in(0,1]$, and $f:[0, \infty) \longrightarrow \mathbb{R}$, let

$$
T_{\alpha}(f)(t)=\lim _{\varepsilon \rightarrow 0} \frac{f\left(t+\varepsilon t^{1-\alpha}\right)-f(t)}{\varepsilon}
$$

for all $t>0, \quad \alpha \in(0,1) . T_{\alpha}$ is called the conformable fractional derivative of $f$ of order $\alpha$.

Definition 1. Let $f^{(\alpha)}(t)$ stands for $T_{\alpha}(f)(t)$. Hence

$$
f^{(\alpha)}(t)=\lim _{\varepsilon \rightarrow 0} \frac{f\left(t+\varepsilon t^{1-\alpha}\right)-f(t)}{\varepsilon} .
$$

If $f$ is $\alpha$-differentiable in some $(0, a), a>0$, and $\lim _{t \rightarrow 0^{+}} f^{(\alpha)}(t)$ exists, then by definition

$$
f^{(\alpha)}(0)=\lim _{t \rightarrow 0^{+}} f^{(\alpha)}(t) .
$$

We should remark that $T_{\alpha}\left(t^{p}\right)=p t^{p-\alpha}$. Further, this definition coincides with the classical definitions of R-L and of Caputo on polynomials (up to a constant multiple).

One can easily show that $T_{\alpha}$ satisfies all the properties in the following theorem: 
Theorem 2. Let $\alpha \in(0,1]$, and $f, g$ be $\alpha$-differentiable at a point $t$. Then:

(1) $T_{\alpha}(a f+b g)=a T_{\alpha}(f)+b T_{\alpha}(g)$, for all $a, b \in \mathbb{R}$.

(2) $T_{\alpha}\left(t^{p}\right)=p t^{p-\alpha}$ for all $p \in \mathbb{R}$.

(3) $T_{\alpha}(f g)=f T_{\alpha}(g)+g T_{\alpha}(f)$.

(4) $T_{\alpha}\left(\frac{f}{g}\right)=\frac{g T_{\alpha}(f)-f T_{\alpha}(g)}{g^{2}}$.

(5) $T_{\alpha}(\lambda)=0$, for all constant functions $f(t)=\lambda$.

(6) If, in addition, $f$ is differentiable, then $T_{\alpha}(f)(t)=t^{1-\alpha} \frac{d f}{d t}$.

Further:

1. $T_{\alpha}\left(t^{p}\right)=p t^{p-\alpha}$ for all $p \in \mathbb{R}$.

2. $T_{\alpha}(1)=0$.

3. $T_{\alpha}\left(e^{c x}\right)=c x^{1-\alpha} e^{c x}, \quad c \in \mathbb{R}$.

4. $T_{\alpha}(\sin b x)=b x^{1-\alpha} \cos b x, \quad b \in \mathbb{R}$.

5. $T_{\alpha}(\cos b x)=-b x^{1-\alpha} \sin b x, \quad b \in \mathbb{R}$.

6. $T_{\alpha}\left(\frac{1}{\alpha} t^{\alpha}\right)=1$

However, it is worth noting the following fractional derivatives of certain functions:

(i) $T_{\alpha}\left(\sin \frac{1}{\alpha} t^{\alpha}\right)=\cos \frac{1}{\alpha} t^{\alpha}$.

(ii) $T_{\alpha}\left(\cos \frac{1}{\alpha} t^{\alpha}\right)=-\sin \frac{1}{\alpha} t^{\alpha}$.

(iii) $T_{\alpha}\left(e^{\frac{1}{\alpha} t^{\alpha}}\right)=e^{\frac{1}{\alpha} t^{\alpha}}$.

\section{The Conformable Fractional Heat Equation}

Let us start with the fact that the second derivative of a function is by definition the iteration of the first derivative. So $y^{\prime \prime}=\frac{d}{d x} \frac{d y}{d x}$.

Hence it is so natural to consider the equation:

$$
\frac{\partial^{\alpha}}{\partial t^{\alpha}} \frac{\partial^{\alpha} u(x, t)}{\partial t^{\alpha}}=\frac{\partial^{2} u(x, t)}{\partial x^{2}}
$$




$$
\begin{aligned}
& u(0, t)=0, \quad t>0, \\
& u(1, t)=0, \quad t>0, \\
& \frac{\partial u(x, 0)}{\partial t}=0, \\
& u(x, 0)=f(x), \quad 0<x<1 .
\end{aligned}
$$

Before we start our discussion, let us discuss conformable fractional linear differential equations with

constant coefficients:

$$
\frac{d^{\alpha}}{d x^{\alpha}} \frac{d^{\alpha} y}{d x^{\alpha}} \pm \mu^{2} y=0
$$

If we associate the auxiliary equation $r^{2} \pm \mu^{2}=0$, to this equation, then the roots are $r= \pm \mu$, or $r= \pm \mu i$. In the first case, we have by formula (iii) above (see [3]) that

$$
y=e^{\mp \frac{\mu}{\alpha} t^{\alpha}}
$$

are two independent solutions of the equation.

In the second case we have by formulas (i) and (ii) above (see [3]), that

$$
y_{1}=\sin \frac{\mu}{\alpha} t^{\alpha} \text { and } y_{2}=\cos \frac{\mu}{\alpha} t^{\alpha}
$$

are two independent solutions of the equation (5).

In general, conformable fractional linear differential equation of order $2 \alpha$, with constant coefficients can be written in the form

$$
\frac{d^{\alpha}}{d x^{\alpha}} \frac{d^{\alpha} y}{d x^{\alpha}}+a \frac{d^{\alpha} y}{d x^{\alpha}}+b y=g(x) .
$$

Let us write $D^{\alpha}$ for $\frac{d^{\alpha}}{d x^{\alpha}}$. So the equation can be written in the form $D^{\alpha}\left(D^{\alpha} y\right)+a D^{\alpha} y+b y=g(x)$. For the homogeneous solution, we consider the equation $r^{2}+a r+b=0$. Using the properties of conformable fractional derivative in [3], and the formulas (i), (ii) and (iii) above one gets the same theory as the usual linear differential equations.

Now we can start discussing our heat equation $(*)$. Let us use separation of variables method.

Let $u(x, t)=P(x) Q(t)$. Substituting in the differential equation to get

$$
\frac{d^{\alpha}}{d t^{\alpha}} \frac{d^{\alpha} Q(t)}{d t^{\alpha}} P(x)=Q(t) \frac{d^{2} P(x)}{d x^{2}},
$$

from which we get

$$
\frac{d^{\alpha}}{d t^{\alpha}} \frac{d^{\alpha} Q(t)}{d t^{\alpha}} / Q(t)=\frac{d^{2} P(x)}{d x^{2}} / P=\lambda,
$$


some constant. Consequently:

$$
\frac{d^{\alpha}}{d t^{\alpha}} \frac{d^{\alpha} Q(t)}{d t^{\alpha}}-\lambda Q=0, \text { and } \frac{d^{2} P(x)}{d x^{2}}-\lambda P=0 .
$$

Now, we consider the equation

$$
\frac{d^{2} P(x)}{d x^{2}}-\lambda P=0 .
$$

As is well known, there are three cases for the values of $\lambda$ to be considered. $\lambda=0, \lambda=-\mu^{2}$ and $\lambda=\mu^{2}$.

Conditions (1) and (2) forces

$$
\mu=n \pi \text { and } P_{n}(x)=c_{n} \sin n \pi x .
$$

Equations (5) and (6), gives

$$
Q(t)=b_{1} \cos \frac{n \pi}{\alpha} t^{\alpha}+b_{2} \sin \frac{n \pi}{\alpha} t^{\alpha}
$$

Condition (3) now gives $b_{2}=0$, and $Q(t)=b_{1} \cos \frac{n \pi}{\alpha} t^{\alpha}$. Consequently, using equation (7), we get

$$
u(x, t)=\sum_{n=1}^{\infty} a_{n} \sin n \pi x \cos \frac{n \pi}{\alpha} t^{\alpha}
$$

Using condition (4) we find that $a_{n}$ is the $n t h$ Fourier coefficient of the function $f(x)$.

One can consider the following form of the conformable fractional differential equation:

$$
\frac{\partial^{\alpha}}{\partial x^{\alpha}} \frac{\partial^{\alpha} u(x, t)}{\partial x^{\alpha}}=\frac{\partial u}{\partial t},
$$

with conditions

$$
\begin{aligned}
& u(x, 0)=f(x), \quad 0<x<1, \\
& u(0, t)=0, \quad t>0, \\
& u(1, t)=0, \quad t>0 .
\end{aligned}
$$

Here equation (5) can be put into action, and one can solve (8) with the given boundary conditions the same way as the ordinary heat equation is solved taking into account formulas (i), (ii) and (iii) above. However, conformable fractional Fourier series comes up in the way. So this will be postponed to a forthcoming paper. 


\section{References}

[1] R. Hilfer, Applications of Fractional Calculus in Physics, World Scientific, Singapore, 2000.

[2] A.A. Kilbas, H.M. Srivastava, and J.J. Trujillo, Theory and Applications of Fractional Differential Equations, Elsevier, Amsterdam, 2006.

[3] R. Khalil , M. Al Horani , A. Yousef , M. Sababheh. Journal of Computational and Applied Mathematics 264 (2014) 65-70.

[4] .K.S. Miller, and B. Ross, An Introduction to the Fractional Calculus and Fractional Differential Equations, A Wiley-Interscience Publication, 1993.

[5] K. Oldham, and J. Spanier. The fractional calculus, theory and applications of differentiation and integration of arbitrary order. Academic Press, U.S.A. 1974 .

[6] I. Podlubny. Fractional differential equations. Academic Press, U.S.A. 1999. 
